\title{
A simple new formulation for phytoplankton photoresponse and an application in a wind-driven mixed-layer model
}

\author{
Peter J. S. Franks ${ }^{1}$, John Marra ${ }^{2}$ \\ ${ }^{1}$ Marine Life Research Group, Scripps Institution of Oceanography - 0218, University of California San Diego, La Jolla, \\ California 92093-0218, USA \\ ${ }^{2}$ Lamont-Doherty Geological Observatory of Columbia University, Palisades, New York 10964, USA
}

\begin{abstract}
We present a simple new formulation of photoresponse in the diatom Lauderia borealis. This formulation assumes time-dependence in the value of $P_{\mathrm{max}}$, the maximal photosynthetic rate. The rate of change of $P_{\max }$ is nonlinearly dependent on the present irradiance, giving a photosynthetic rate that depends on the weighted light history of the cell. The 2 new parameters introduced in this formulation were found to be robust, i.e. they did not change for a variety of data sets. The photoresponse model was incorporated into a simple Lagrangian model of wind-driven vertical mixing. It was found that the modelled photoresponse can lead to enhanced vertically integrated photosynthesis under certain conditions of light extinction and wind mixing. In particular, phytoplankton in wind-mixed coastal regions may experience an increase in integrated photosynthesis per cell under strong incident irradiance.
\end{abstract}

KEY WORDS: Photoresponse $\cdot$ Phytoplankton $\cdot$ Mixing

\section{INTRODUCTION}

The measurement of primary production in the ocean typically involves collecting a series of water samples over the depth of the euphotic zone, filling bottles with the water samples, and incubating them, either back in the ocean suspended from a buoy or on the deck of a ship, under conditions which simulate the temperature and light of the depth from which they were collected. After incubation, the samples are analyzed for the determinants of photosynthesis, such as changes in dissolved oxygen or the assimilation of ${ }^{14} \mathrm{C}$ into particulate matter. The samples are usually incubated over the course of the daylight period, or else $24 \mathrm{~h}$, to integrate over diurnal variability in solar irradiance, and to obtain a number reflecting the daily rate of primary production.

This time period is long with respect to changes in phytoplankton physiology (e.g. Harris \& Lott 1973, Marra 1980), motion in the upper ocean (Denman \&
Gargett 1983, Weller et al. 1985), and local meteorology (Price et al. 1986). A long incubation of a series of bottles suspended at discrete depths may, therefore, only approximate the true rate of production by the phytoplankton in the freely circulating water of the mixed layer. Decreasing the incubation period, on the other hand, requires extrapolation to diurnal rates with an unknown precision, both physiologically and environmentally.

Partly because of the move toward basin, if not global, scales for the estimation of primary production, and because of the need to develop relationships among environmental forcing, production, and export of that production to the deep sea, the methods by which primary production is measured and modelled continue to be of compelling interest. Despite gains made in the evaluation of in vitro methodology, however, the fidelity of in vitro rates in representing real rates of photosynthesis in mixed layers remains unknown. Incubations impose a stratified water col- 
umn on the measurement, creating inaccuracies in situations with vertical mixing of the water.

The problem is 2-fold: vertical mixing combined with changes in incident irradiance imply that phytoplankton cells in the same water column all have different light histories. Second, there are physiological responses to the changes in irradiance creating temporal variability in the photosynthetic response to local irradiance. For the latter, while the first-order model which relates photosynthesis to irradiance is well described (Jassby \& Platt 1976), the factors that regulate temporal changes of the model parameters, i.e. phytoplankton photoresponse, are not well understood. We present here a model that describes the nature of phytoplankton photoresponse to rapidly changing irradiance, characteristic of oceanic mixed layers and diurnal timescales. We first derive the photoresponse model, and constrain the 2 new parameters with experimental data. We then incorporate the photoresponse model into a simple model of winddriven vertical mixing to examine the effects of photoresponse on vertically integrated photosynthesis under a range of environmental conditions.

\section{PHOTORESPONSE MODEL}

\section{The model}

The photoresponse model developed here is based on the experiments of Marra (1978a, b), in which an oxygen electrode measured photosynthetically generated oxygen in a culture of Lauderia borealis under various irradiance regimes. It was noted from these experiments that: (1) cell-specific photosynthetic oxygen output decreased with time under constant irradiance, (2) the rate of decrease of oxygen output was high for high light, and lower for low light, and (3) the $P$-I relationship at any given time was a saturating response.

It is these features which were considered essential for the photoresponse model to recreate. The modelling approach is empirical: we seek to describe the net cell-specific oxygen output of a cell under the influence of fluctuating irradiance, without describing the details of the physiological mechanisms creating that output.

The model for photoresponse is based on a framework common to many $P$ - $I$ models: a saturating response of photosynthesis to increasing irradiance (e.g. Jassby \& Platt 1976). Indeed, the basic model structure is very similar to that of Neale \& Marra (1985), with some important distinctions which will be noted below. We began with a basic saturating $P$-I relationship, the hyperbolic tangent (Jassby \& Platt 1976):

$$
P(I)=P_{\max } \tanh \left(\alpha I / P_{\max }\right)
$$

where $P(I)$ is the irradiance-dependent photosynthetic oxygen output (pg-at. $\mathrm{O}_{2}$ cell $^{-1} \mathrm{~h}^{-1}$ ), $P_{\max }$ is the maximal photosynthetic output (pg-at. $\mathrm{O}_{2}$ cell $^{-1} \mathrm{~h}^{-1}$ ), $\alpha$ is the initial slope of the $P$ - $I$ curve Ipg-at. $\mathrm{O}_{2}$ cell $^{-1} \mathrm{~h}^{-1}$ $\left.\left(\mu \text { Einstein } \mathrm{m}^{-2} \mathrm{~s}^{-1}\right)^{-1}\right]$, and $I$ the irradiance ( $\mu$ Einstein $\left.\mathrm{m}^{-2} \mathrm{~s}^{-1}\right)$. We then assumed $P_{\max }$ to be time dependent (sensu Neale \& Marra 1985, Pahl-Wostl \& Imboden 1990) with a rate of change dependent on its present value, and the present irradiance (as suggested by Cullen \& Lewis 1988). Contrary to Neale \& Marra (1985), however, we assumed the rate of change of $P_{\max }$ to be nonlinearly related to the present irradiance. We used $P_{t}$ to represent the time-dependent $P_{\max }$ (same units), and assumed first-order kinetics to govern the response to irradiance shifts (e.g. Falkowski \& Wirick 1981, Lewis et al. 1984a, Neale \& Marra 1985, Cullen \& Lewis 1988). A general formulation for the rate of change of $P_{t}$ is then:

$$
\begin{aligned}
\frac{\mathrm{d} P_{t}}{\mathrm{~d} t}=\frac{\mathrm{d}}{\mathrm{d} t} P_{\max }(I, t) & =-\frac{1}{\delta}\left(\frac{I}{I_{0}}\right)^{1 / a} P_{t} \\
& =-\frac{I^{1 / a}}{\gamma} P_{t} .
\end{aligned}
$$

Two new parameters were introduced in this formulation for the time-dependent $P_{\max }$ : $\gamma$ and a. The parameter $\gamma\left(=\delta I_{0}{ }^{1 / a}\right)$ represents a timescale for the photoresponse, while $a$ is a parameter controlling the degree of nonlinearity of the rate response to light intensity. The units of $\gamma\left[\left(\mu \text { Einstein } \mathrm{m}^{-2} \mathrm{~s}^{-1}\right)^{1 / a} \mathrm{~h}\right]$ depend on the choice of the nondimensional number $a$, and the units of time (in this case, hours). The parameters $I_{0}$ and $\delta$ scale the irradiance and time, respectively. The relationship of $\gamma$ to $\delta$ and $I_{0}$ will be discussed below.

This formulation of the time-dependence of $P_{\max }$ assumes that $P_{t}$ tends toward zero after long times. A more general formulation could include an asymptote for $P_{t}, P_{0}$ :

$$
\frac{\mathrm{d} P_{t}}{\mathrm{~d} t}=\frac{\mathrm{d}}{\mathrm{d} t} P_{\max }(I, t)=\frac{I^{1 / \mathrm{a}}}{\gamma}\left(P_{0}-P_{t}\right) .
$$

It was found during fitting of the model to the data that $P_{0}$ was very small. In addition, the small reduction in residuals obtained using a model with $P_{0}$ did not justify the inclusion of the extra parameter.

For an arbitrary time-dependent irradiance, $I(t)$, a general solution to $\mathrm{Eq}$. (2) is:

$$
P_{t}(I, t)=P_{\max } \exp \left(-\frac{1}{\gamma} \int_{0}^{t} I^{1 / a} \mathrm{~d} t^{\prime}\right)
$$

where $P_{\max }\left(=P_{t}\right.$ at $\left.t=0\right)$ is the constant of integration, 
and $t$ the time since dawn. The integral in this equation is an integral of the irradiance encountered by the cell over time. This equation indicates that $P_{l}$ is dependent on the weighted irradiance history of the cell, the weighting depending on the time and the irradiance itself. For a constant light intensity, Eq. (4) reduces to

$$
P_{l}(I, t)=P_{\max } \exp \left(-\frac{I^{1 / a}}{\gamma} t\right)
$$

The full time-dependent photosynthesis-irradiance response involves substitution of $P_{t}$ [either Eq. (4) or Eq. (5)] for $P_{\max }$ in Eq. (1):

$$
P(I, t)=P_{l} \tanh \left(\alpha I / P_{t}\right) \text {. }
$$

The behavior of both the time-dependent (Eqs. $4 \& 6$ ) and steady (Eqs, 5 \& 6) photoresponse formulations will be explored below.

The general formulation of the model (Eqs. $3 \& 6$ ) allows $P_{t}$ to increase during a light period if $P_{t}<P_{0}$. The specific formulation used here (Eqs. 4, $5 \& 6$ ) sets $P_{0}=0$, so that $P_{t}$ always decreases with time. This is consistent with the data upon which the model was based, except for 1 experiment at a very low light level (Fig. 1), but limits the application of the model to 1 diurnal period. For simulations $>1 \mathrm{~d}, P_{t}$ would have to be reset to $P_{\max }$ during the dark period. This behavior is consistent with the observation that many diatoms from temperate latitudes grow better under a

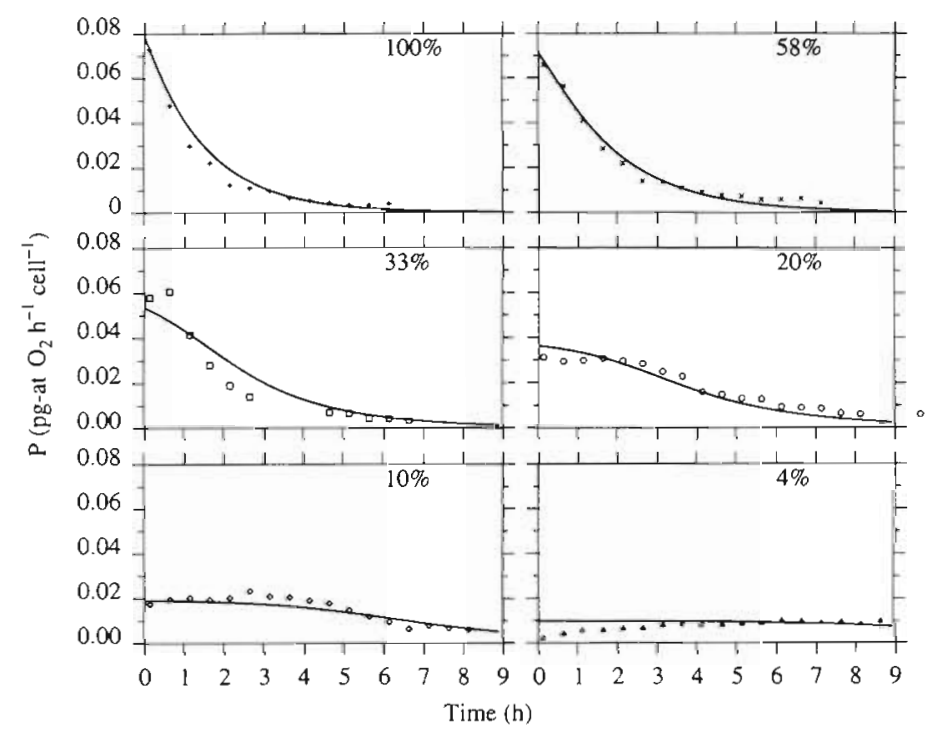

Fig. 1. Lauderia borealis. Symbols: time-dependent oxygen production per cell under 6 steady irradiances. $100 \%=1500 \mu$ Einstein $\mathrm{m}^{-2}$ $\mathrm{s}^{-1}$. Solid lines: model simulations (Eqs. $5 \& 6$ ) of the data. $P_{\max }=$ $0.08 \mathrm{pg}$-at. $\mathrm{O}_{2}$ cell $^{-1} \mathrm{~h}^{-1}, \alpha=1.3 \times 10^{-4} \mathrm{pg}$-at. $\mathrm{O}_{2}$ cell $^{-1} \mathrm{~h}^{-1}(\mu$ Einstein $\left.\mathrm{m}^{-2} \mathrm{~s}^{-1}\right)^{-1}, \quad \gamma=17.1$ ( $\mu$ Einstein $\left.\mathrm{m}^{-2} \mathrm{~s}^{-1}\right)^{1 / 3} \mathrm{~h}, a=3$. Data from Marra (1978b) light/dark cycle than under constant illumination (Brand \& Guillard 1981). Long-term ( $>1$ d) changes in $P_{\max }$ could be incorporated into this model as the value to which $P_{t}$ is reset each dark period.

\section{Parameter estimation}

The model developed here has 4 parameters: $P_{\max }$, $\alpha, \gamma$ and $a$. To estimate these parameters we used nonlinear least-squares minimization of the combined residual for all data sets to fit the steady model (Eqs. 5 $\& 6$ ) to the data of Marra (1978b; his Fig. 2) (Fig. 1). The parameter a controls the nonlinearity of the response of $P_{t}$ to light intensity. The model was fairly insensitive to changes in $a$ when $2.5<a<3$.5. It was found that $a=3$ was quite robust, working equally well for all data sets (see below) and having the pleasing attribute of being an integer. If $a=3$, the parameter $\gamma$ has units $\left[\left(\mu \text { Einstein } \mathrm{m}^{-2} \mathrm{~s}^{-1}\right)^{1 / 3} \mathrm{~h}\right]$

The model was more sensitive to changes in $\gamma_{1}$ which was found to be constant for all but 1 data set and irradiance condition. The best fit to the data of Marra (1978b, his Fig. 2, and 1978a, his Figs. 3a, 4a \& 5a) was $\gamma=17.1\left(\mu \text { Einstein } \mathrm{m}^{-2} \mathrm{~s}^{-1}\right)^{1 / 3} \mathrm{~h}$. The odd units of $\gamma$ suggest that it may be a combination of 2 parameters; one scaling the irradiance response, the other the temporal response. Following Eq. (2) we can write

$$
\gamma=\delta\left(I_{0}\right)^{1 / a}
$$

where $\delta(h)$ scales the time and $I_{0}\left(\mu\right.$ Einstein $\mathrm{m}^{-2}$ $\mathrm{s}^{-1}$ ) scales the irradiance. Based on Marra's (1978a, b) data an appropriate value for $I_{0}$ is $1500 \mu$ Einstein $\mathrm{m}^{-2} \mathrm{~s}^{-1}$ (the maximal irradiance), thus $\delta$ is $1.5 \mathrm{~h}$. The specific response timescale from Eq. (2), $I^{1 / d} / \gamma$, ranges from zero at low irradiances to $0.7 \mathrm{~h}^{-1}$ at $1500 \mu$ Einstein $\mathrm{m}^{-2} \mathrm{~s}^{-1}$ (Fig. 2).

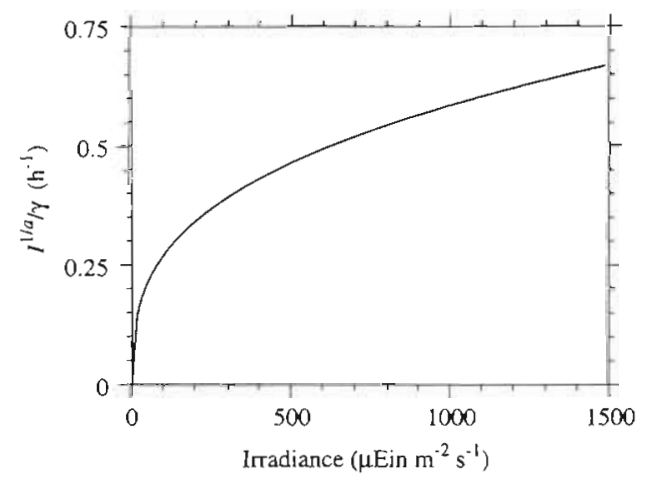

Fig. 2. Lauderia borealis. Specific photoresponse timescale, $I^{1 / 3} / \gamma$, obtained from Eq. (2), as a function of irradiance. $\gamma=17.1\left(\mu \text { Einstein } \mathrm{m}^{-2} \mathrm{~s}^{-1}\right)^{1 / 3} \mathrm{~h}$ 

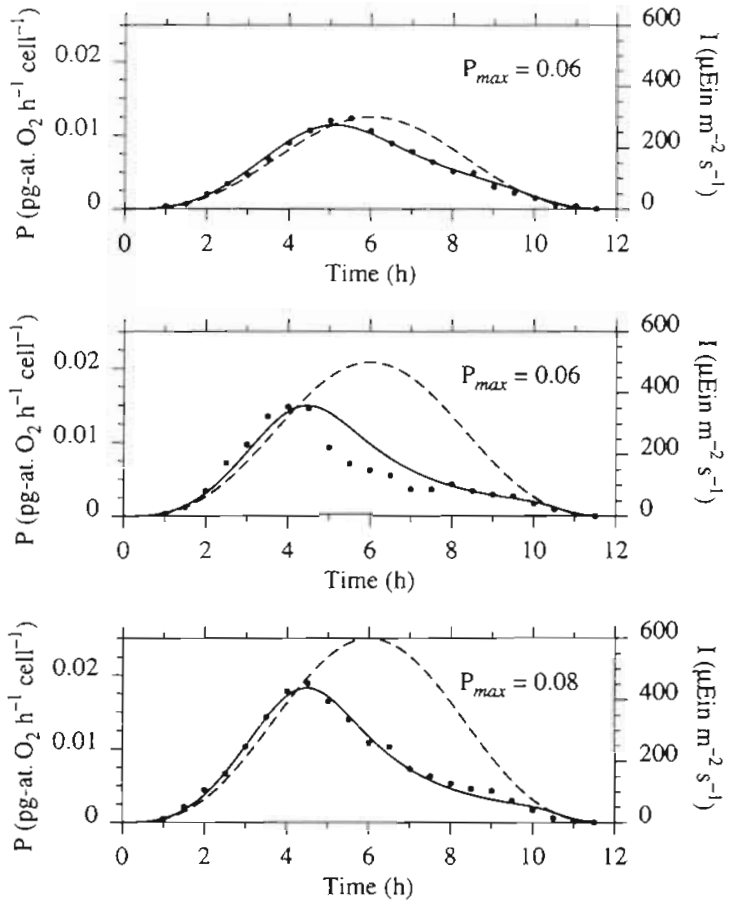

Fig. 3. Lauderia borealis. Symbols: time-dependent oxygen production under temporally varying irradiance. Solid lines: fit of model (Eqs. $4 \&$ 6) to the data. $\alpha=0.5 \times 10^{-4} \mathrm{pg}$-at. $\mathrm{O}_{2}$ cell ${ }^{-1} \mathrm{~h}^{-1}\left(\mu \text { Einstein } \mathrm{m}^{-2} \mathrm{~s}^{-1}\right)^{-1}, \gamma=17.1\left(\mu \text { Einstein } \mathrm{m}^{-2} \mathrm{~s}^{-1}\right)^{1 / 3} \mathrm{~h}$

$a=3$. Dashed lines: irradiance. Data from Marra (1978a)

The main features of the photoresponse of Lauderia borealis to steady light were reproduced by this model (Fig. 1): the photosynthetic oxygen output decreased with time; the rate of decrease was slow for low light and fast for high light; and the shape of the oxygen evolution curves changed from concave upward for high irradiance to initially concave downward for low irradiance. The same values for $P_{\max }$ and $\alpha$ could be used for all experiments, even though the experiments were conducted over a series of days (6-11 January 1977), indicating the robustness of this model.

Using the values for $\gamma$ and $a$ found above, the model (Eqs. 4 \& 6) was fit to data from experiments with temporal variations in irradiance (Marra 1978a; Fig. 3). An analytical function (sensu Marra 1978a) was used to simulate the irradiance field,

$$
I(t)=I_{\max } \sin ^{3}\left(\frac{\pi t}{12}\right),
$$

where $I_{\max }$ is the maximal irradiance over a $12 \mathrm{~h}$ daylight period. The model fits to these data were excellent; the post-noon depression of photosynthesis was reproduced accurately, as was the increasingly nonsymmetric photosynthetic output over the day with higher peak irradiances. It was found that the best fit

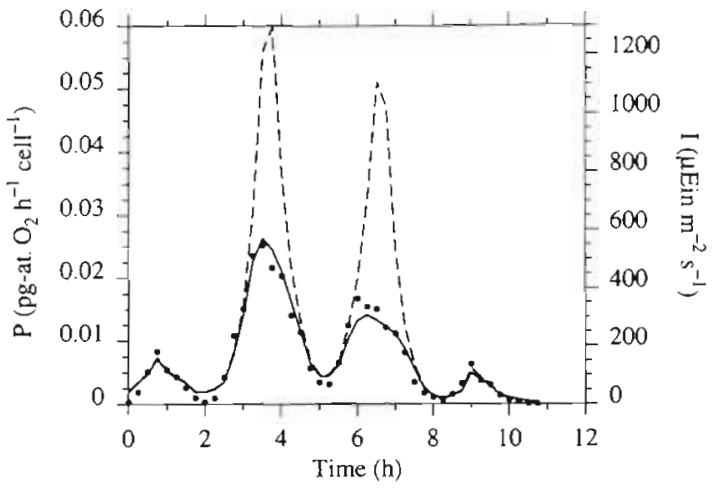

Fig. 4. Lauderia borealis. Symbols: time-dependent oxygen production under temporally varying irradiance. Solid lines: fit of model (Eqs. $4 \& 6$ ) to the data. Dashed lines: irradiance. $P_{\max }=0.052 \mathrm{pg}$-at. $\mathrm{O}_{2}$ cell $^{-1} \mathrm{~h}^{-1}, \alpha=0.45 \times 10^{-4}$ pg-at. $\mathrm{O}_{2}$ cell ${ }^{-1} \mathrm{~h}^{-1}\left(\mu \text { Einstein } \mathrm{m}^{-2} \mathrm{~s}^{-1}\right)^{-1}, \gamma=30.4\left(\mu \text { Einstein } \mathrm{m}^{-2} \mathrm{~s}^{-1}\right)^{1 / 3} \mathrm{~h}$, $a=3$

to the data was obtained with a slightly higher $P_{\max }$ for the $I_{\max }=600 \mu$ Einstein $\mathrm{m}^{-2} \mathrm{~s}^{-1}$ case than the other 2 cases. Otherwise the parameters were identical for all experiments. Given that the experiments were performed over a period of $2 \mathrm{mo}$, some drift in the culture parameters would be expected.

The model was also used to describe the photosynthetic oxygen output from an experiment with fluctuating irradiance (Marra 1978a, his Fig. 4b; present Fig. 4). The best fit was obtained with $\gamma=30.4$ ( $\mu$ Einstein $\left.\mathrm{m}^{-2} \mathrm{~s}^{-1}\right)^{1 / 3} \mathrm{~h}$, or equivalently, $\delta=2.6 \mathrm{~h}$ when $I_{0}=1500$ $\mu$ Einstein $\mathrm{m}^{-2} \mathrm{~s}^{-1}$, giving a specific response timescale of $0.38 \mathrm{~h}^{-1}$ at $I=1500 \mu$ Einstein $\mathrm{m}^{-2} \mathrm{~s}^{-1}$.

The fits of the time-dependent photoresponse model to the data showed the 2 new parameters, $\gamma$ and $a$, to be quite robust. The value $a=3$ allowed good fits of the model to all data sets, while $\gamma$ varied from 17 to $30\left(\mu \text { Einstein } \mathrm{m}^{-2} \mathrm{~s}^{-1}\right)^{1 / 3} \mathrm{~h}$ (or $1.5<\delta<2.6 \mathrm{~h}$ ). The model accurately reproduced most of the nonlinear features of the experiments, from the nonlinear relationship of the rate of change of $P_{t}$ to light intensity, to the variably asymmetric diumal photosynthetic oxygen output under temporally varying light.

An important feature of the model was its indication that the present photosynthetic output of the cell was a nonlinear function of the weighted irradiance history since dawn. This implies that cells moving independently in a surface mixed layer could have significantly different photosynthetic output at the same present irradiance level, due to differences in their past irradiance. This variability could have important implications for the integrated photosynthetic output of a population in a surface mixed layer. To explore the effects of photoresponse in a wind-forced mixing layer a Lagrangian model of time-dependent mixing and photoresponse was developed. 


\section{LAGRANGIAN MIXING MODEL}

The 1-dimensional (vertical) mixing-layer model was based on the Lagrangian formulation of eddy diffusivity developed by Yamazaki \& Kamykowski (1991). We follow Yamazaki \& Kamykowski's notation of $K_{D}$ for the Lagrangian eddy diffusivity to distinguish it from the Eulerian diffusivity, $K_{z}$. Briefly, a spatially dependent eddy diffusivity, $K_{D}$, was calculated to depend on the dissipation of turbulent kinetic energy, $\varepsilon$, the kinematic viscosity, $v$, and the buoyancy frequency, $N$ :

$$
K_{D}=2.7 \varepsilon^{1 / 2} v^{1 / 2} N^{-1} \text {. }
$$

The dissipation of kinetic energy was assumed to be related to a friction velocity, u., von Karman's constant, $\kappa \approx 0.4$, and depth, $z$ :

$$
\varepsilon(z)=u_{s}^{3} / \kappa z
$$

Finally $u$. was related to the surface wind stress, $\tau$, and the water density, $\rho$, by

$$
u_{.}=(\tau / \rho)^{1 / 2}
$$

We followed Yamazaki \& Kamykowski's (1991) choice of parameters, using $N=0.001 \mathrm{~s}^{-1}, \rho=1025 \mathrm{~kg} \mathrm{~m}^{-3}$, and $v=1 \times 10^{-6} \mathrm{~m}^{2} \mathrm{~s}^{-1}$. The Lagrangian diffusivity, $K_{D}$, was then used to calculate the local standard deviation of the step size of a vertical random walk, $\left(2 K_{D} \Delta t\right)^{1 / 2}$, where $\Delta t$ is the temporal step size (180 s). The potential step size thus decreased with depth, and increased with surface wind stress. Reflecting boundary conditions were used at the surface and depth of an assumed pycnocline $(15 \mathrm{~m})$. Thus cells within the mixing layer were moved vertically, whereas cells below the mixing layer were stationary. The $1 / z$ dependence of $\varepsilon$ allows $\varepsilon$ to be unbounded when $z<1$. To alleviate this problem, we set $\varepsilon(z<1)=\varepsilon(z=1)$.

The surface irradiance field was as specified above (Eq. 8), with a peak irradiance, $I_{\max }$, of $1500 \mu$ Einstein $\mathrm{m}^{-2}$ $\mathrm{s}^{-1}$ at noon. To specify the underwater light intensity, a constant light extinction coefficient, $k_{\text {ext }}$, was used:

$$
I(z)=I(0) \exp \left(-k_{\mathrm{ext}} z\right)
$$

Since there were important interactions between the depth of mixing and the euphotic depth (assumed here to be the $1 \%$ light level), a variety of extinction coefficients were explored under several surface wind stresses and mixing-layer depths.

The instantaneous photosynthetic output of 2000 cells, initially distributed evenly throughout the water column, was followed over $1 \mathrm{~d}$ (Figs. 5 \& 6) for 2 different wind stresses ( $\tau=0$ and $0.2 \mathrm{~Pa}$ ) and 2 different extinction coefficients $\left(k_{\mathrm{ext}}=0.3\right.$ and $\left.0.1 \mathrm{~m}^{-1}\right)$. Since the cases with no mixing $(\tau=0 \mathrm{~Pa})$ are identical except for the extinction coefficient, depths have been scaled
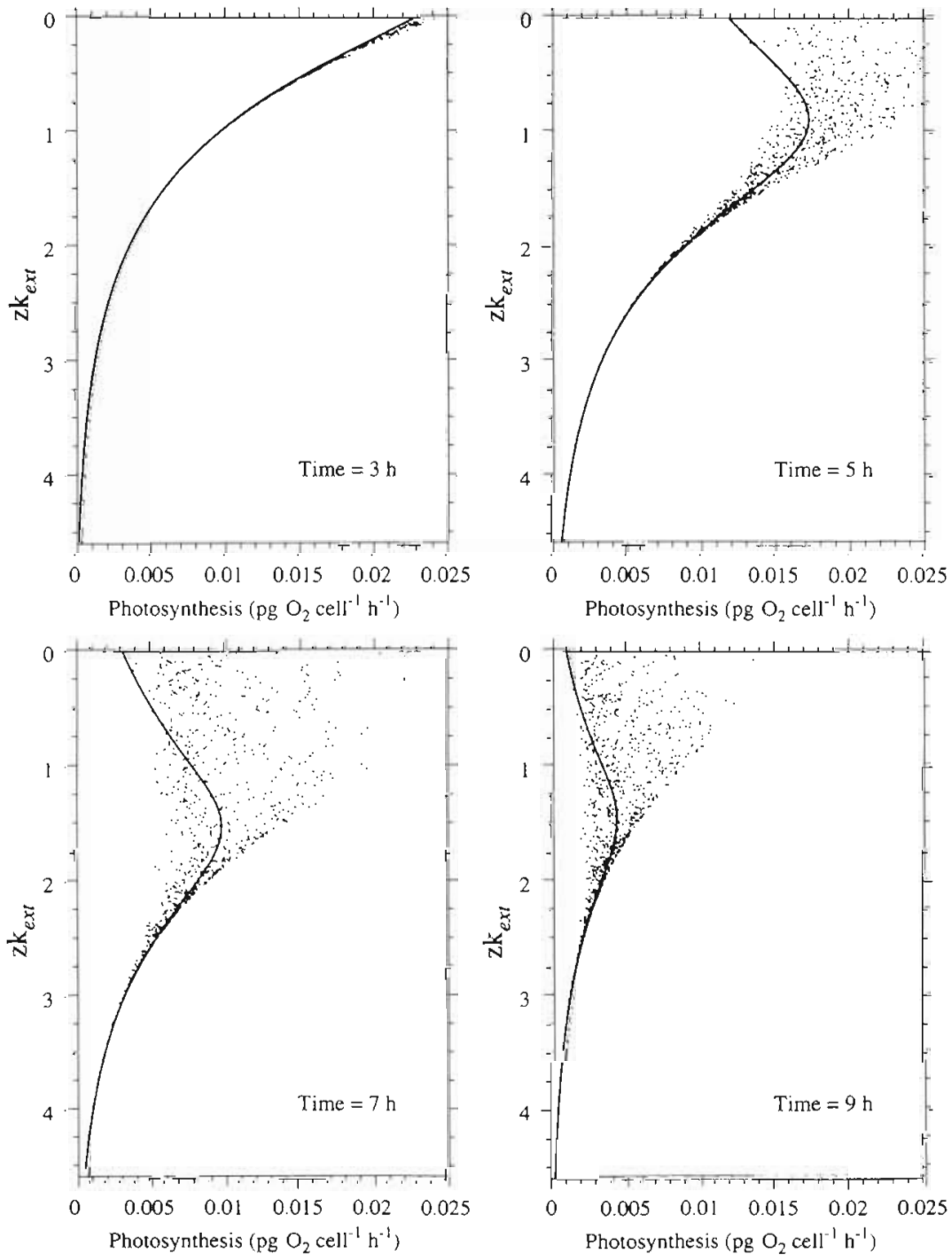

Fig. 5. Lauderia borealis. Vertical distribution of instantaneous photosynthetic rate of 2000 cells under calm (solid line) and wind-mixed ( $\tau=0.2 \mathrm{~Pa}_{\text {; dots) }}$ conditions, at 4 times after dawn for $k_{\text {ext }}=0.3 \mathrm{~m}^{-1}$. Other parameters: $P_{\max }=$ 0.08 pg-at. $\mathrm{O}_{2}$ cell $^{-1} \mathrm{~h}^{-1}, \alpha=0.5 \times 10^{-4} \mathrm{pg}$-at. $\mathrm{O}_{2}$ cell $^{-1} \mathrm{~h}^{-1}\left(\mu \text { Einstein } \mathrm{m}^{-2} \mathrm{~s}^{-1}\right)^{-1}$. $\gamma=17.1\left(\mu \text { Einstein } \mathrm{m}^{-2} \mathrm{~s}^{-1}\right)^{1 / 3} \mathrm{~h}, a=3, I_{\max }=1500 \mu$ Einstein $\mathrm{m}^{-2} \mathrm{~s}^{-1}$ Mixinglayer depth is $15 \mathrm{~m}$ 

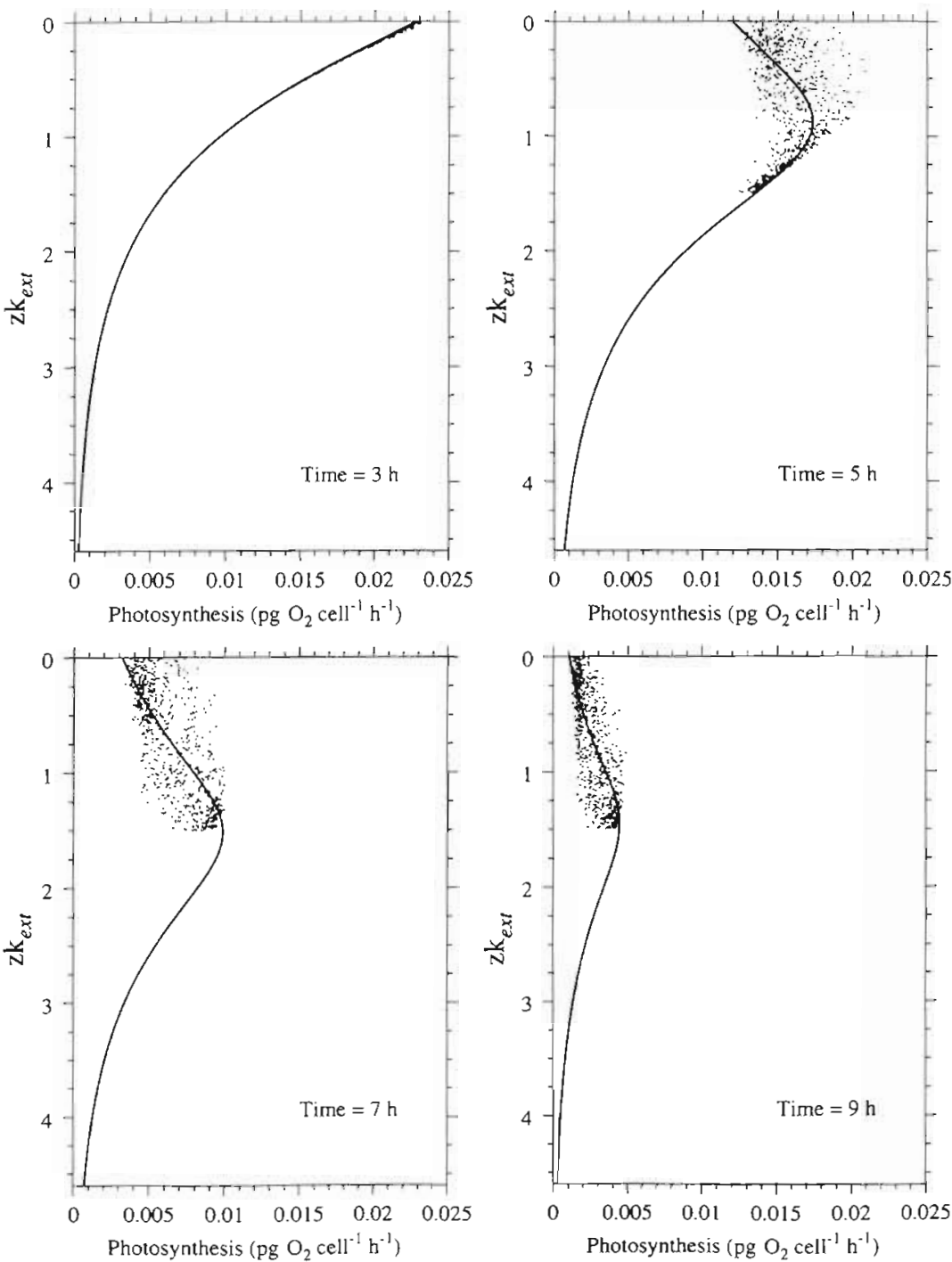

Fig. 6. Lauderia borealis. Vertical distribution of instantaneous photosynthetic rate. As Fig. 5, except $k_{\text {ext }}=0.1 \mathrm{~m}^{-1}$

by $k_{\text {ext }}$ for ease of comparison between cases. Thus the mixing-layer depth $(15 \mathrm{~m})$ appears at a scaled depth of 4.5 for $k_{\text {ext }}=0.3 \mathrm{~m}^{-1}(0.3 \times 15=4.5)$, and 1.5 for $k_{\text {ext }}=$ $0.1 \mathrm{~m}^{-1}$. The cells in the high $k_{\text {ex }}$ case were mixed through a much larger light gradient than the low $k_{\text {ext }}$ case. This was reflected in the variability of the instantaneous photosynthetic output at a given depth: the higher variability in the $k_{\mathrm{ext}}=0.3 \mathrm{~m}^{-1}$ case reflected the higher variability in light histories of the cells as they were mixed to and from a given optical depth.

The photosynthetic output at a given depth could be higher in the mixed case than the nonmixed case as cells from deeper (lower irradiance) waters were mixed upward. Because of the former low light levels, these cells had not accumulated as much irradiance as cells nearer the surface, allowing them to have higher values of $P_{t}$. The exponentially decreasing envelope of maximum phatosynthesis with depth in the high $k_{\text {ext }}$ mixed case reflected photosynthesis on the light-limited portion of the $P$-I curve. In the mixed case there were fewer cells with a low $P_{t}$ thus fewer cells experienced saturating light. The mixing thus ameliorated the effects of near-surface photoresponse. Again, this was less effective in the case with low $k_{\text {ext }}$ as the cells were not mixed from as great an optical depth, thus the cells had more similar light histories.

These results were reflected in the vertical distribution of $P_{l}$ (Figs. $7 \& 8$ ). The mixing created significant variability in $P_{l}$ at a given depth, with higher variability in the high $k_{\text {ext }}$ case. On average $P_{t}$ was increased at the surface, and decreased at depth by mixing, these changes being more substantial when $k_{\text {ext }}$ was large. The decrease of $P_{t}$ at depth was less significant to the actual level of photosynthesis, since the light level was low there and photosynthesis was light limited. At the surface, however, the increased values of $P_{t}$ created an enhanced photosynthesis, on average. The variability of $P_{t}$ thus resulted in variability of photosynthesis near the surface, but had little effect on photosynthesis below -2.5 optical depths $\left(k_{\mathrm{ext}} z>2.5\right)$ where photosynthesis was light limited.

The effects of various wind stresses on the vertically integrated photosynthetic output per cell were examined by comparing the mixed and unmixed cases (Fig. 9). In general, as the wind stress increased, the vertically integrated photosynthesis per cell increased. The increase was more pronounced with a higher $k_{\text {ext }}$.

The increase of integrated photosynthesis with wind stress also depended on the depth of the mixing layer (Fig. 10). For a given wind stress, a deeper mixing layer gave an increased integrated photosynthesis, this increase being most noticeable in the high $k_{\text {ext }}$ case. Very little change in the integrated photosynthesis was seen when $k_{\text {ext }}=0.08 \mathrm{~m}^{-1}$, while increases up to $50 \%$ occurred when $k_{\text {ext }}=0.3 \mathrm{~m}^{-1}$. The increases were quite steep for $k_{\text {ext }}=0.3 \mathrm{~m}^{-1}$, with significant changes at a wind stress of $0.1 \mathrm{~Pa}$ and a mixing-layer depth of $10 \mathrm{~m}$. The changes of inte- 

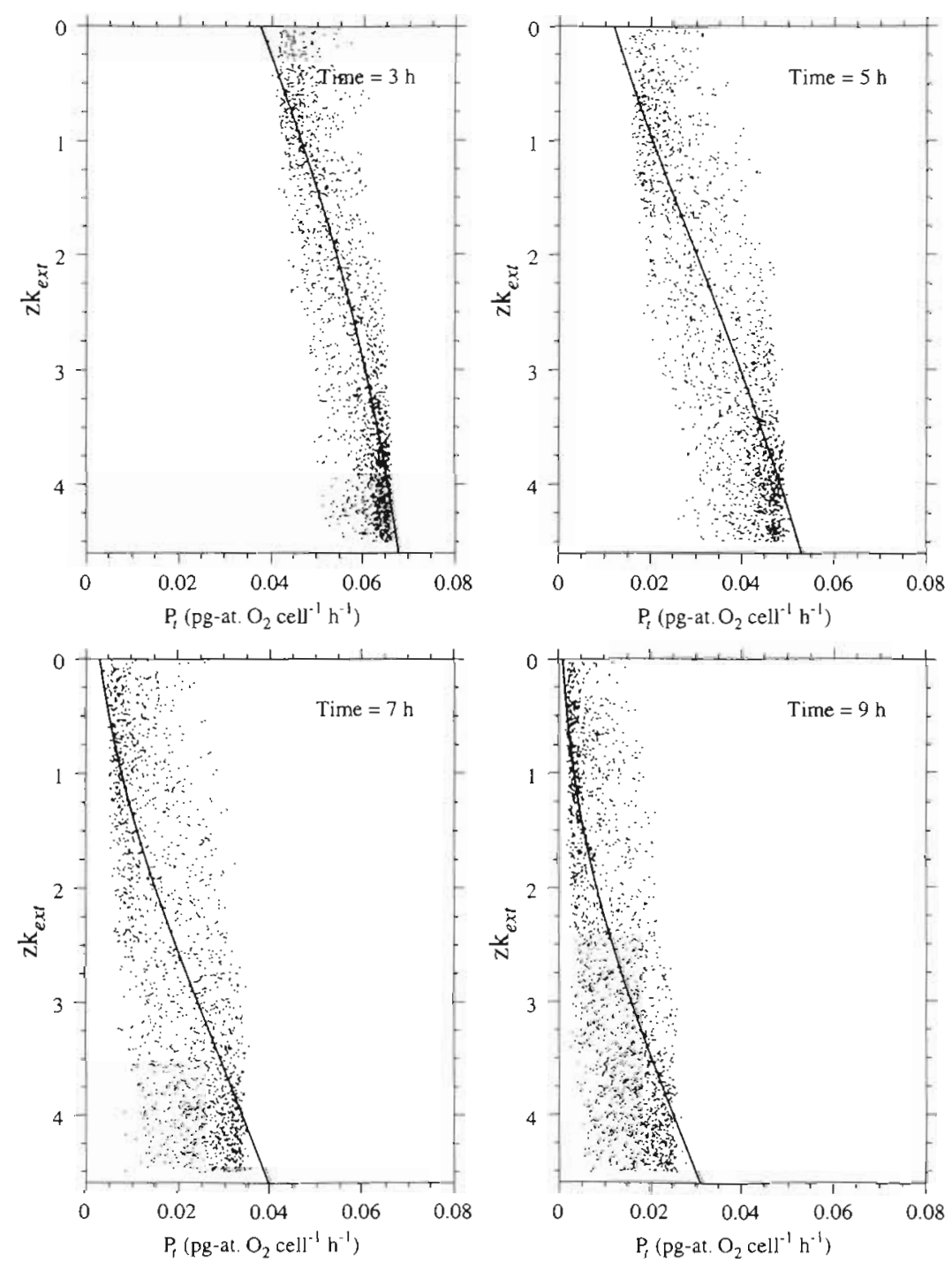

Fig. 7. Lauderia borealis. Vertical distribution of $P_{t}$ of 2000 cells under calm (solid line) and wind-mixed ( $\tau=0.2 \mathrm{~Pa}$; dots) conditions, at 4 times after dawn for $k_{\mathrm{ext}}=0.3 \mathrm{~m}^{-1}$. Other parameters as in Fig. 5

grated photosynthesis with mixing-layer depth had maxima, beyond which the integrated photosynthesis decreased. The location of these maxima depended on the time at which the profile was taken, the mixing-layer depth and the wind stress.

\section{DISCUSSION}

A time-dependent photoresponse model was presented which assumed the temporal variability of photosynthesis to result from changes in a temporally varying $P_{\text {max }}$, denoted $P_{t}$. The rate of change of $P_{t}$ was modelled as a nonlinear function of the light intensity and a first-order relation to the present $P_{t}$. The equa- tion for the present photosynthetic rate was found to depend on the weighted light history of the cell. Two new parameters, $\gamma$ and $a$, were introduced in this formulation. In a 1-dimensional vertical mixing model, the photoresponse model allowed increased vertically integrated photosynthesis when mixed by the wind, this response being strongest when the light extinction coefficient was high $\left(k_{\text {ext }}=0.3 \mathrm{~m}^{-1}\right)$. Increased wind stress gave increased integrated photosynthesis for a given mixing-layer depth, while integrated photosynthesis increased with mixing-layer depth for a given wind stress. These responses were always more pronounced for water columns with a high $k_{\text {ext }}$.

The photoresponse model presented here is similar in many respects to other models in the literature. The use of first-order kinetics to describe the temporal changes of $P_{\text {max }}$ is common (e.g. Falkowski \& Wirick 1981, Lewis et al. 1984a, Neale \& Richerson 1987 , Cullen \& Lewis 1988). However, this formulation combining first-order kinetics and a nonlinear response to light intensity is unique. The simple formulation has the advantages that it requires only 2 new parameters, both of which appear to be quite robust, and it is analytic, and so can easily be incorporated into models of photosynthesis. Compared to other models based on the same data sets (Neale \& Marra 1985, Denman \& Marra 1986, Pahl-Wostl \& Imboden 1990), the present model requires fewer parameters and is simpler to implement, yet gives as accurate a fit to the data.

The parameters $a$ and $\gamma$ were found to be fairly robust for the data sets used to constrain the model. It is likely, however, that these parameters are not only time-dependent, but also species-dependent. The nearly $2 \times$ variation of $\gamma$ among the experimental treatments is an indication that these parameters are not constant. However we have no way, presently, of assessing the degree of variation to be expected in these parameters. The parameters of this model were chosen based on experiments with Lauderia borealis, and are probably not valid for other phytoplanktonic species. It is likely that strong latitudinal gradients exist in $a$ and $\gamma_{1}$ since phytoplankton at high latitudes are 

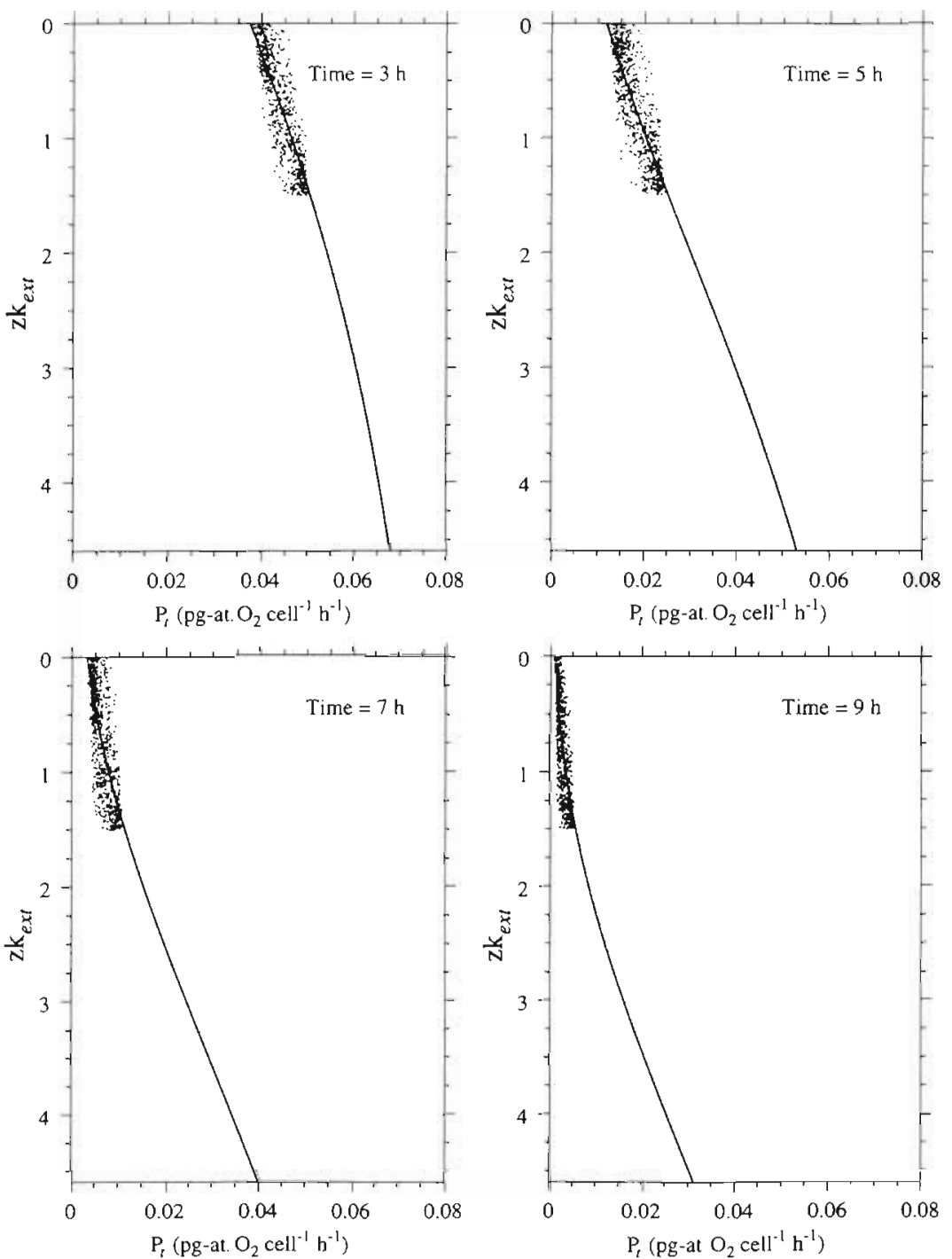

Fig. 8. Lauderia borealis. Vertical distribution of $P_{1}$. As Fig. 7, except $k_{\text {ext }}=0.1 \mathrm{~m}^{-1}$

It was not necessary to posit any timedependence of $\alpha$ in the present model; excellent fits of the model to the data were obtained with only variation of $P_{\max }$. Models including temporal changes in $\alpha$ did not give improved fits to the data, and required 3 new parameters. It is possible that a larger dynamic range of changes in $P_{\max }$ overwhelm variations in photosynthetic output caused by changes in $\alpha$.

The present model includes a range of timescales for the response of photosynthesis to changes in the irradiance field. The time response is scaled by $I^{1 / a} / \gamma$, giving a specific rate of change of $P_{\max }$ from zero at low light to $0.7 \mathrm{~h}^{-1}$ at $1500 \mu$ Einstein $\mathrm{m}^{-2} \mathrm{~s}^{-1}$. This range includes almost all the values found by Cullen \& Lewis (1988) for response of $P_{\max }$ to various light changes, supporting the suggestion that the rate of change is nonlinearly related to the present irradiance.

The increase in vertically integrated photosynthesis caused by wind mixing of a photoresponding phytoplankton population supports the hypothesis that photoresponse can be an important mechanism to exploit turbulent motions within the mixing layer (Harris \& Lott 1973, Marra 1978a, Gallegos \& Platt 1982, Lewis et al. 1984a, b, Lande \& Lewis 1989). Gallegos \& Platt (1985) define a dimensionless variable, $t^{*}=$ $2 k_{\text {ext }}^{2} K_{Z} t$, which represents (for the present case) the time since dawn scaled by the time required for a pop-

often exposed to constant irradiance. A photoresponse following the present model would be disadvantageous under such conditions. Alternatively, there could be latitudinal gradients in $P_{0}$, the asymptotic photosynthetic rate (Eq. 3). At temperate latitudes, the photoperiod is short enough relative to the response timescale that $P_{l}$ never reaches $P_{0}$ (Fig. $1_{i} P_{0}=0$ ), thus $P_{0}=0$ does not affect integrated daily photosynthetic output. At higher latitudes with longer photoperiods, $P_{0}>0$ would represent a background photosynthetic rate toward which $P_{t}$ tends, allowing for increases in $P_{t}$ if $P_{t}<P_{0}$

In the present model we considered only timedependence of $P_{\max }$. Time-dependence of $\alpha$ is known (e.g. Lewis et al. 1984a, Cullen \& Lewis 1988), and the rate constants for response are similar to those for $P_{\max }$. ulation of phytoplankton to be mixed vertically through 1 optical depth. Using $K_{D}$ (Eq. 9) rather than $K_{Z}$, we can evaluate $t^{*}$ for the mixing experiments described above. It was found that a fairly linear relationship exists $\left(\mathrm{R}^{2}=0.91\right)$ between the ratio of vertically integrated photosynthesis from mixed and nonmixed cases, and $t^{\bullet}$ (Fig. 11). Further analyses confirmed the linear dependence of the enhancement ratio on incubation time, and the exponential dependence on light extinction coefficient. The relationship is weak at small values of $t^{*}$ (low extinction coefficient, short time since dawn, low wind stress), which may account for the equivocal results obtained from laboratory and field data. Based on these results, we would expect the most consistent enhancement of integrated photosynthesis in coastal regions (high $k_{\text {exc }}$ ) 


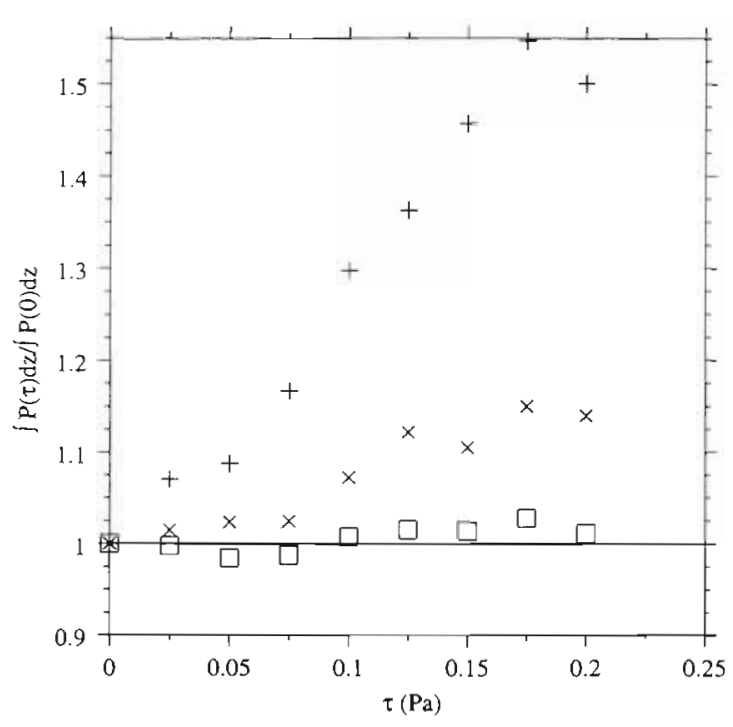

Fig. 9. Lauderia borealis. Vertically integrated photosynthesis per cell at $9 \mathrm{~h}$ after dawn: ratio of mixed $[P(\tau)]$ to unmixed $[P(0)] . k_{\text {ext }}=0.3 \mathrm{~m}^{-1}(+), 0.15 \mathrm{~m}^{-1}(\times)$, or $0.08 \mathrm{~m}^{-1}(\square)$. Other parameters as in Fig. 5

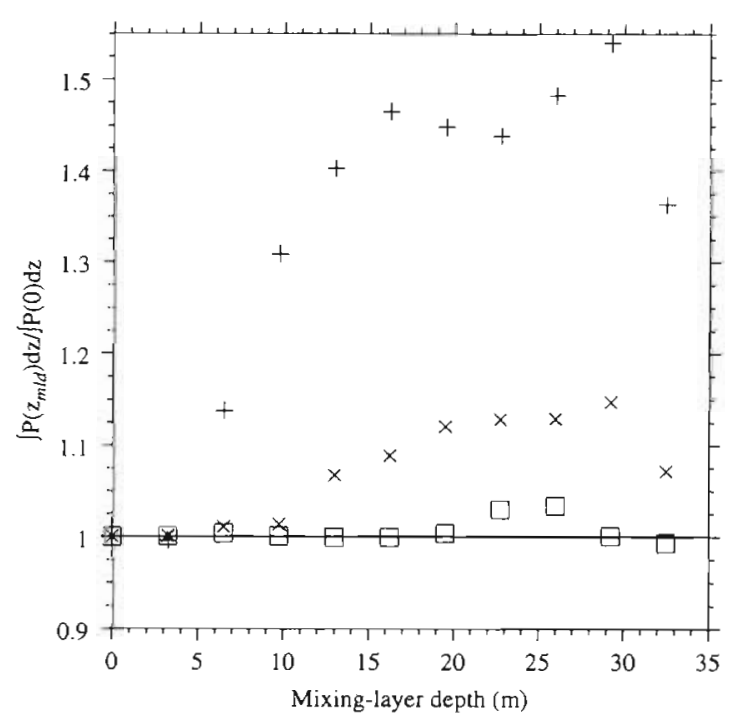

Fig. 10. Lauderia borealis. Vertically integrated photosynthesis per cell at $9 \mathrm{~h}$ after dawn: ratio of case with mixing-layer depth $\left[\mathrm{P}\left(z_{\text {mid }}\right)\right]$ to no mixing-layer depth $[\mathrm{P}(0)]$. Symbols as in Fig. 9

under strong winds. This prediction was not borne out in field experiments by Yoder \& Bishop (1985), who found enhancement of photosynthesis in only 5 of 47 bottles, with $t^{*}$ values between 10 and 500 . They attributed this lack of enhancement to a lack of photoinhibition under high irradiance (see also Gallegos \& Platt 1985)

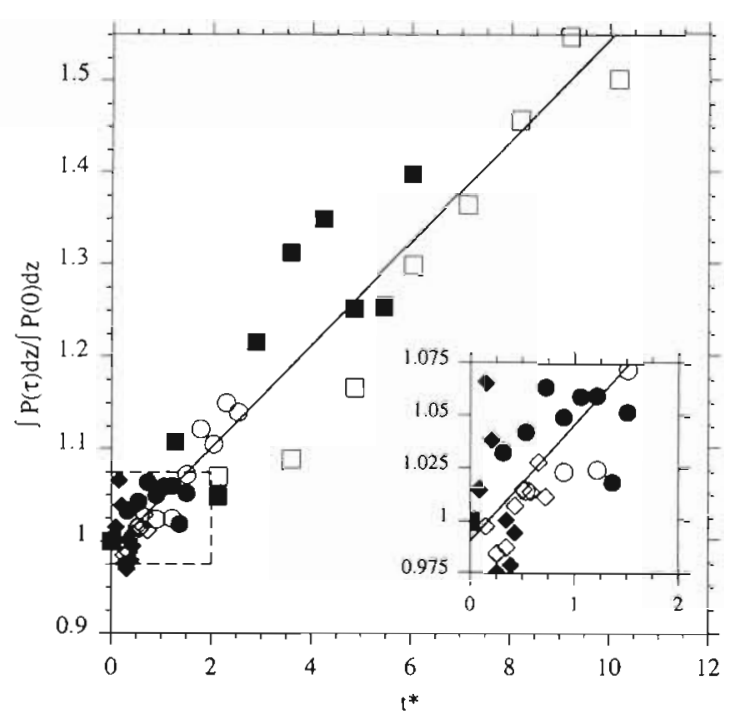

Fig. 11 Lauderia borealis. Relationship of $t^{*}=2 k_{\text {ext }} K_{D} t$ to enhancement ratio of mixed to nonmixed phytoplankton populations. Open symbols: $15 \mathrm{~m}$ mixing-layer depth; filled symbols: $30 \mathrm{~m}$ mixing-layer depth. $k_{\text {ext }}=0.3 \mathrm{~m}^{-1}(\square, \mathbf{\square})$, $0.15 \mathrm{~m}^{-1}(0, \bullet)$, or $0.08 \mathrm{~m}^{-1}(\diamond, \bullet)$. Other parameters as in Fig. 5

In the absence of mixing, the photoresponse model presented here creates a vertical profile which has a subsurface peak in photosynthesis (Figs. $5 \& 6$, solid lines). The decrease of photosynthesis at the surface is a result of photoresponse to the high light within the upper 1.5 optical depths. Photosynthesis in this region is saturated under unmixed conditions, forcing low values of $P_{t}$ under the present model. Below 1.5 optical depths, photosynthesis is light limited, giving an exponential decrease with depth even in the mixing case. The effect of mixing is to redistribute cells within the mixing layer; if the mixing layer extends below 1.5 optical depths, the integrated photosynthesis is increased (Fig. 10; mixing-layer depths $>1.5 / k_{\text {ext }}$ ), as high $P_{t}$ cells are mixed into the upper euphotic zone.

The strength of the wind mixing influences the length of time a given cell experiences a given irradiance. At higher wind stresses, the cells are exposed to high light for shorter periods, resulting in higher average $P_{t}$ in the upper euphotic zone (Figs. $7 \& 8$ ). This results in an increasing integrated photosynthesis with increasing wind stress (Fig. 9). The present model ignores the interaction of wind stress and mixing-layer depth, assuming the latter to be stationary throughout a single day. While this assumption may be approximately valid for a diurnal period, over several days the mixing layer would increase. This increase in the depth of the turbulent mixing layer could couple with the photoresponsive mechanisms of the phytoplankton to generate additional increases in the integrated photosynthesis. 


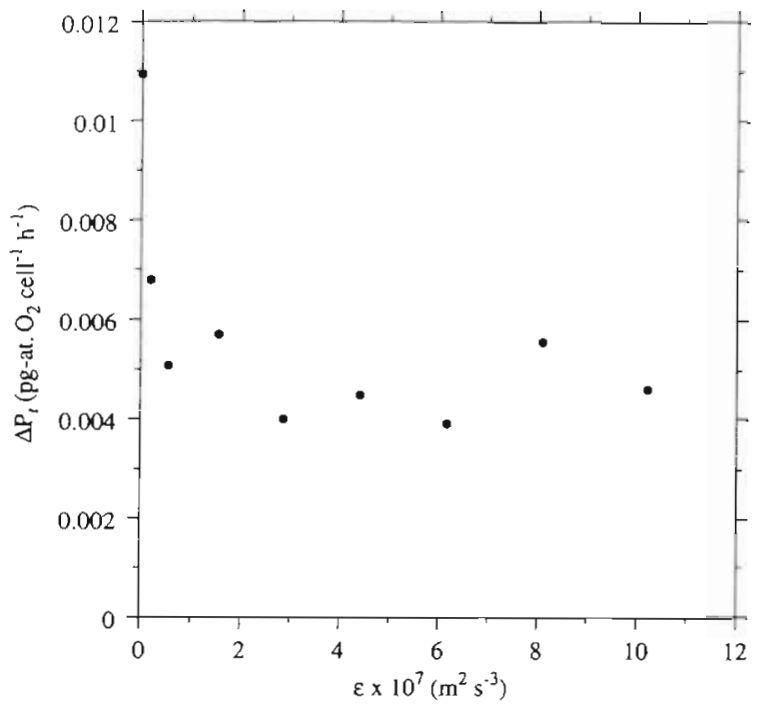

Fig. 12. Lauderia borealis. Difference between average $P_{t}$ in $1 \mathrm{~m}$ depth interval centered at $1 \mathrm{~m}$ and $P_{t}$ in $1 \mathrm{~m}$ depth interval centered at $1 / k_{\text {ext }}$ for $k_{\text {ext }}=0.3 \mathrm{~m}^{-1}$, over a range of wind-induced turbulent energy dissipation rates, $\varepsilon$. Other parameters as in Fig. 5

The surface irradiance field used was an extreme case in the sense that it would cause the greatest photoresponse; lower irradiances would cause smaller changes in $P_{t}$. Higher frequency changes in incident light, for example those caused by clouds, would ultimately cause less variation of $P_{t}$ and a less pronounced change in integrated photosynthesis. These results are consistent with some of the experimental results of Marra (1978b), Gallegos \& Platt (1982) and Marra et al. (1985), in which it was found that mixing sometimes had little effect on the integrated production. In particular, photosynthesis on the light-limited portion of the $P$-I curve resulted in highly predictable integrated photosynthesis. The present model suggests that these results arise from the low rate of change of $P_{t}$ at low irradiances (Eq. 2).

Theory on the vertical distribution of photoresponsive parameters (e.g. Lewis et al. 1984a, b, Cullen \& Lewis 1988) suggests that parameters with a response timescale longer than the mixing timescale should be vertically homogeneous in a well-mixed water column. Results from the present model support this result to a degree (Fig. 12). When $\Delta P_{t}$, the difference between the average $P_{l}$ at the surface and the first attenuation depth, is plotted versus dissipation of turbulent kinetic energy, $\varepsilon$ (sensu Lewis et al. 1984b; their Fig. 2), a nonlinear decrease of $\Delta P_{t}$ with $\varepsilon$ is seen. While the dynamic range of $\Delta P_{t}$ in the present model is much smaller than that of Lewis et al. (1984b), the patterns are similar. However, the present model never achieves complete homogenization of $P_{t}$, and $\Delta P_{t}$ is time dependent (Figs. $7 \&$ 8). Differences between the present model and Lewis et al. (1984a) arise from the nonlinear dependence of $P_{t}$ on irradiance, the lack of a steady state in the present model, and a depth-dependent eddy diffusivity, $K_{D}$ (Yamazaki \& Kamykowski 1991). The present model suggests that a better indication of the effects of mixing on photosynthesis might be to somehow measure the variability of $P_{t}$ at a given depth (e.g. Lande \& Lewis 1989). In particular, the variance and skewmess of the distribution of $P_{t}$ at a given depth should increase as mixing increases. With the present model, $P_{t}$ is positively skewed at the surface, and negatively skewed at depth by vertical mixing (Figs. 7 $\& 8$ ), the degree of skewness changing with time. The mean $P_{t}$, therefore, is not a very good representation of the average photosynthetic rate of the population at a given depth. In particular, it underestimates the photosynthesis at the surface under mixed conditions

Acknowledgements. This paper is funded in part by a grant to P.J.S.F. from the National Oceanic and Atmospheric Administration. The views expressed herein are those of the authors and do not necessarily reflect the views of NOAA or any of its subagencies. Funding to J.M. was through ONR contract number N00014-89-J-1150. This is contribution 18 of the U.S. GLOBEC program, funded jointly by NOAA and NSF.

\section{LITERATURE CITED}

Brand, L. E., Guillard, R. R. L. (1981). The effects of continuous light and light intensity on the reproduction rates of twenty-two species of marine phytoplankton. J. exp. mar. Biol. Ecol. 50: 119-132

Cullen, J. J., Lewis, M. R. (1988). The kinetics of algal photoadaptation in the context of vertical mixing. J. Plankton Res. 10: 1039-1063

Denman, K. L., Gargett, A. E. (1983). Time and space scales of vertical mixing and advection of phytoplankton in the upper ocean. Limnol. Oceanogr. 28: 801-815

Denman, K. L., Marra, J. (1986). Modelling the time dependent photoadaptation of phytoplankton to fluctuating light. In: Nihoul, J. C. J. (ed.) Marine interfaces ecohydrodynamics. Elsevier, Amsterdam, p. 341-349

Falkowski, P. G., Wirick, C. D. (1981). A simulation model of the effects of vertical mixing on primary productivity. Mar. Biol. 65: 69-75

Gallegos, C. L., Platt, T. (1982). Phytoplankton production and water motion in surface mixed layers. Deep Sea Res. 29: $65-76$

Gallegos, C. L., Platt, T (1985). Vertical advection of phytoplankton and productivity estimates: a dimensional analysis. Mar. Ecol. Prog. Ser. 26: 125-134

Harris, G. P., Lott, J. (1973). Light intensity and photosynthetic rates in phytoplankton. J. Fish. Res. Bd Can. 30 $1771-1778$

Jassby, A. D., Platt, T. (1976). Mathematical formulation of the relationship between photosynthesis and light for phytoplankton. Limnol. Oceanogr. 21: 540-547

Lande, R., Lewis, M. R. (1989). Models of photoadaptation and 
photosynthesis by algal cells in a turbulent mixed layer Deep Sea Res. 36: 1161-1175

Lewis, M. R., Cullen, J. J., Platt, T (1984a). Relationships between vertical mixing and photoadaptation of phytoplankton: similarity criteria. Mar. Ecol. Prog. Ser. 15 $141-149$

Lewis, M. R., Horne, E. P. W., Cullen, J. J., Oakey, N. S., Platt, $\mathrm{T}$ (1984b). Turbulent motions may control phytoplankton photosynthesis in the upper ocean. Nature 311:49-50

Marra, J. (1978a). Effect of short-term variations in light intensity on photosynthesis of a marine phytoplankter: a laboratory simulation study. Mar. Biol. 46: 191-202

Marra, J. (1978b). Phytoplankton photosynthetic response to vertical movement in a mixed layer. Mar. Biol. 46: 203-208

Marra, J. (1980). Vertical mixing in primary production. In Falkowski, P. (ed.) Primary productivity in the sea. Plenum, New York, p. 121-137

Marra, J., Heinemann, K., Landriau, G. Jr (1985). Observed and predicted measurements of photosynthesis in a phytoplankton culture exposed to natural irradiance. Mar. Ecol. Prog. Ser. 24: 43-50

Neale, P., Marra, J. (1985). Short-term variation of $P_{\max }$ under

This article was presented by M. R. Lewis, Halifax, N.S., Canada natural irradiance conditions: a model and its implications. Mar. Ecol. Prog. Ser. 26: 113-124

Neale, P. J., Richerson, P. J. (1987). Photoinhibition and the diurnal variation of phytoplankton photosynthesis - I. Development of a photosynthesis-irradiance model from studies of in situ responses. J. Plankton Res. 9: 1647-1693

Pahl-Wostl, C., Imboden, D. M. (1990). DYPHORA - a dynamic model for the rate of photosynthesis of algae. J. Plankton Res. 12: 1207-1221

Price, J. F., Weller, R. A., Pinkel, R. (1986). Diurnal cycling: observations and models of the upper ocean response to diurnal heating. J. geophys. Res. 91: 8411-8427

Weller, R. A., Dean, J. P., Marra, J., Price, J. F., Francis, E. A., Boardman, D. C. (1985). Three dimensional flow in the upper ocean. Science 227: 1552-1556

Yamazaki, H., Kamykowski, D. (1991). The vertical trajectories of motile phytoplankton in a wind-mixed water column. Deep Sea Res. 38: 219-241

Yoder, J. A., Bishop, S. S. (1985). Effects of mixing-induced irradiance fluctuations on photosynthesis of natural assemblages of coastal phytoplankton. Mar. Biol. 90: $87-93$

Manuscript first received: September 15, 1993

Revised version accepted: April 19, 1994 